\title{
Model Based Analysis of Error Recursive Method for Self-Regulation Processes
}

\author{
P. Aravind ${ }^{1 *}$ and S. M. Girirajkumar ${ }^{2}$ \\ 1'Faculty of Electrical Engineering, Anna University, Chennai - 600025, Tamil Nadu, India; venkyaravind@gmail.com \\ 2Department of Instrumentation and Control Engineering, Saranathan College of Engineering, \\ Tiruchirappalli -620012,Tamil Nadu, India; smgirirrajkumar@gmail.com
}

\begin{abstract}
Objectives: To improve the closed loop response by means of reducing the rise time, settling time and overshoots by implementing a suitable method. Methods/Statistical Analysis: Three different higher order transfer functions has been considered for analysis and are approximated to First Order Plus Dead Time models (FOPDT) using process reaction curve method by applying unit step change. Particle Swarm Optimization technique; and Internal Model Control (IMC) method are used to tune Proportional, Integral and Derivative (PID) control values. Findings: The new method, Error Recursive Reduction Computational (ERRC) method has been tested on higher order systems. The proposed method ERRC is merged with PSO based PID and IMC based PID controllers in hybrid form to form new control structure. Comparative analysis was carried out between with ERRC and without ERRC. Application/Improvements: It can be applied to feedback control system i.e., feedback control applications are highly influenced in chemical, manufacturing automobile industries and etc.
\end{abstract}

Keywords: Higher Orders Systems, Modeling, PID Control, Recursive Analysis, Self Regulating Process

\section{Introduction}

Proportional Integral Derivative (PID) controller is the most well-known control scheme that has been broadly actualized all through the industrial process for as far back as decades. These control parameter settings are obtained by various tuning techniques such as being ZieglerNichols (Z-N) method, the Cohen-Coon method ${ }^{1-3}$, Model based PID control (IMC) $)^{4}$, optimization algorithms $s^{5}$ and so on. In order to show signs of improvement in closed loop execution an alternate controller setting for every operation locale must be utilized $\frac{6,7}{}$.

The goal of the study is to compare tuned PID control setting based on Particle Swarm Intelligence (PSO) optimization algorithm and conventional method with proposed method. Hence, this work summarizes the simplicity of ERRC method procedures to obtain the best result. Here, higher order systems are approximated to first order plus dead time transfer function using process reaction curve method. The three parameters are identified are process gain $(\mathrm{K})$, time constant $(\mathrm{t})$, and dead time $\left(t_{d}\right)$. The performance of the proposed control method in this article is tested by simulations on (approximated FOPTD models) several higher order linear systems with different characteristics.

It is compared against a PSO based and IMC based PID. Implementation configuration is and also its robustness is checked. No need to include literature survey as separate title. Introduction should represent the background of the recent advancements on the problem of your study and should convey how your study is warranted in the existing context. In other words, what is the need for your study and relevance of it? You may also state whether your study is first of its kind or to verify the existing claim or hypothesis. Also highlight how your study is going to link the existing gaps on such problem. You may also clearly mention the objectives of your study. 


\section{Controller Design}

In this segment, the propose of PSO based PID controller, ERRC tuning formulas and implementation procedures are discussed.

\subsection{PSO Based PID Controller}

PSO is used to find the best fitted values PI control settings. It was developed in 1995 by James Kennedy (social-psychologist) and Russell Eberhart (electrical engineer). New solutions are chosen with regards to their fitness; the extra proper they're the extra probabilities. The higher than is repetitive till the condition is satisfied. The error criterion, the time integral absolute value of error (ITAE) is taken as the objective function; it's thus one in the entire premier creating controller institutionalization strategies.

\subsection{Error Recursion-Reduction Computational Technique}

The fundamental idea behind this system is to restrict the error in order to pick up an immaculate control activity, in an incorporated way with the controller. All things considered, an error is found out on the relationship exists between real output (yp) and target output (tp),

Error, $\mathrm{E}=\left(\mathrm{t}_{\mathrm{p}}-\mathrm{y}_{\mathrm{p}}\right)$

The physical equation is examined about the present process output $\left(\mathrm{P}_{\mathrm{i}}\right)$ and respective error $\left(\mathrm{e}_{\mathrm{i}}\right)$, prediction of the output $\left(\mathrm{P}_{\mathrm{i}+1}\right), \mathrm{i}=1,2,3,4 \ldots \ldots$ and error $\left(\mathrm{e}_{\mathrm{i}}\right)$, $\mathrm{i}=1,2,3,4 \ldots$.

Output, $P_{i+1}=\left[P_{i-1}-t_{p}+P_{i-1}\right]$

The estimation of error is done dynamically and minimized.

The goal of the structuring control framework is to keep up the process variable at set value a sensor is utilized to measure, process factors from the plant. For example, consider the controlled variable and sensor is equivalent. Subsequently considering $\mathrm{c}(\mathrm{t})$ as controlled variable and $y(t)$ as sensor output. At that point, the sensor output will be, $y(t)=c(t)$. The computational calculation of ERRC system has been talked about in two cases:, as follows, In case 1 , If the sensor output $(y(t))$ is greater than the reference input $(\mathrm{r}(\mathrm{t}))$ and the error $\mathrm{e}(\mathrm{t})$ is calculated as a difference between sensor output and reference input, $r(t)$. The output from the ERRC $(m(t))$, is obtained by adding an error value with sensor output, which gives a fitted value to meet a system demands. In case 2, consider the sensor output is lesser than reference value $r(t)$ and by subtracting sensor output from reference input and adding a compensating parameter is $(t)$ to give an output of ERRC.

Take $y_{c}(t)=y(t)$ during the computation process for the betterment of closed loop response, detail procedure is created in the flow chart shown in Figure 1 and 2 is the implementation of two control techniques. This technique can be implemented in any kind of process, irrespective to order of the system.

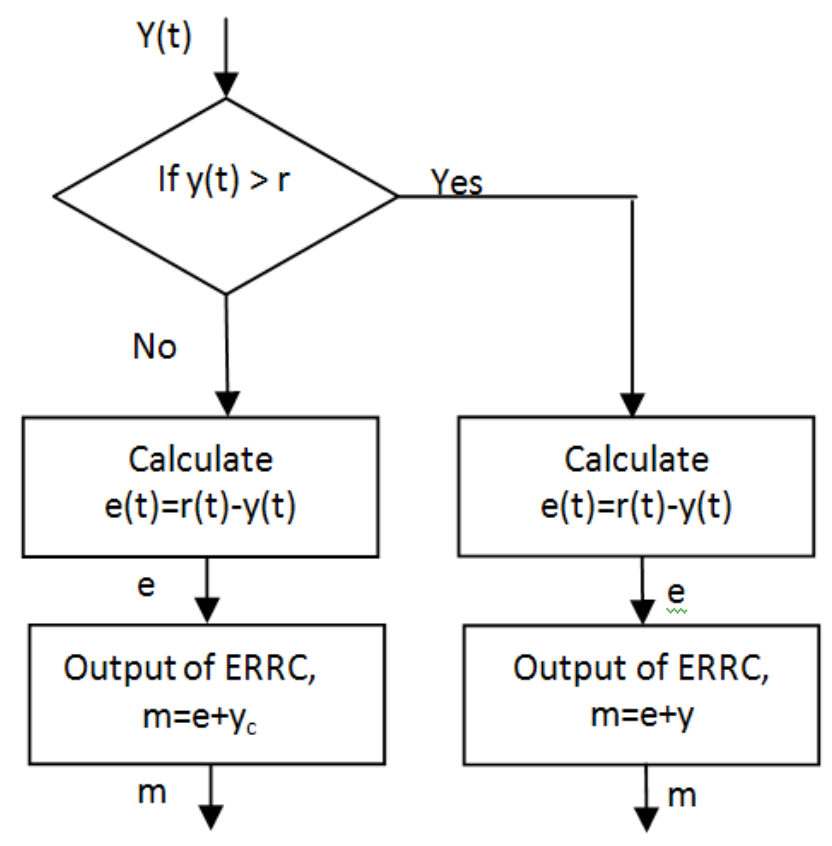

Figure 1. Parameter estimation process.

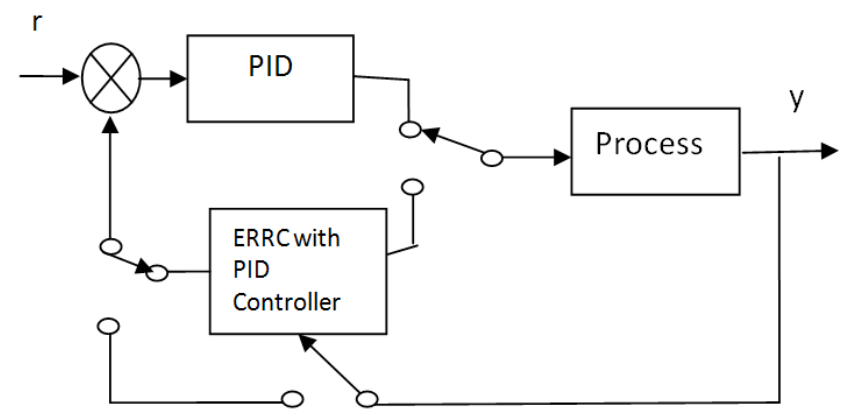

Figure 2. Implementation of ERRC and PID controller closed loop system. 


\section{Results and Comparison}

In this section, to evaluate the performance and robustness of the proposed method is compared with the responses of the original design (PSO based PID), the alternative design with ERRC (ERRC+PID) and PSOPID controller.

Different linear higher order systems are defined by the following transfer functions, were used for testing the proposed approach.

$$
\begin{aligned}
& G_{11}(S)=\frac{(s+3)}{(s+1)(s+2)} * e^{-2 s} \\
& G_{22}(S)=\frac{10(s+2)(s+4)}{(s+1)(s+3)(s+5)} * e^{-3 s} \\
& G_{33}(S)=\frac{1}{(s+1)(s+3)(2 s+1)(5 s+1)} * e^{-0.5 s}
\end{aligned}
$$

The above system transfer functions are approximated to FOPTD model as follows by process reaction curve method as $G_{1}(2), G_{2}(s), G_{3}(s)$ respectively.

$$
G(S)=\frac{K}{(T s+1)} * e^{-T_{d} s}
$$

The first system is a second order system ; second system is a third order system and third system is a fifth order system with different dead time and different time constantis shown in Figure 3 and transfer function parameters are presented in Table 1 .

Table 1. Transfer function parameters

\begin{tabular}{|l|l|l|l|l|}
\hline \multirow{2}{*}{$\begin{array}{l}\text { S. } \\
\text { No. }\end{array}$} & \multirow{2}{*}{ System } & \multicolumn{3}{|l|}{ Parameters } \\
\cline { 3 - 5 } & & $\mathbf{K}$ & $\mathbf{T}$ & $\mathbf{T}_{\mathbf{d}}$ \\
\hline 1 & $\mathrm{G}_{1}(\mathrm{~s})$ & 1.5 & 1.215 & 1.62 \\
\hline 2 & $\mathrm{G}_{2}(\mathrm{~s})$ & 5.33 & 3.9 & 2.83 \\
\hline 3 & $\mathrm{G}_{3}(\mathrm{~s})$ & 0.333 & 9.2 & 0.7 \\
\hline
\end{tabular}

\subsection{Tuning of Control}

For system, $G_{1}(s)$, PSO is used for finding values for PID parameters and for $G_{2}(s) G_{3}(s)$, internal model control method is used to tune PID parameters ${ }^{8}$.

Tuned PID values are presented in Table 2. The design strategy of PSO based PID controller is described in past segment. In this section, the end decisive factor is thought to be the fulfillment of worthy wellness esteem design which happlens with the 100 cycles $^{6}$. In every cycle the finest among the 100 particles taken as potential result are picked.

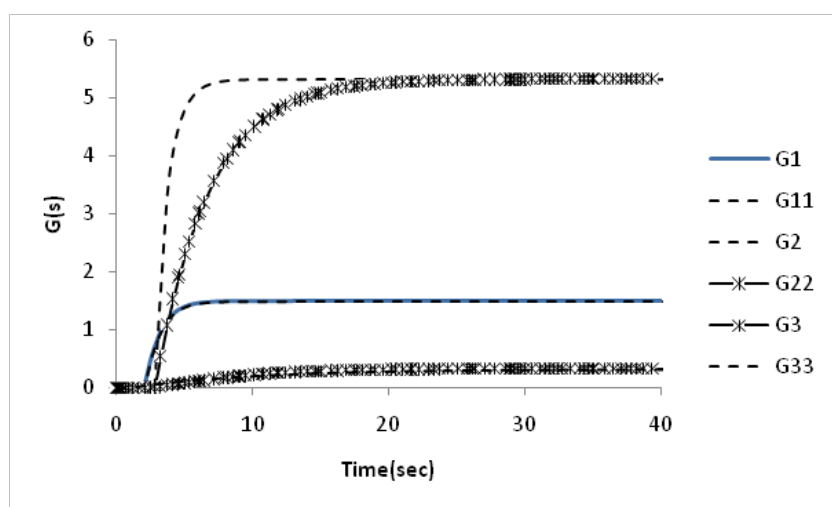

Figure 3. Open loop response of higher order systems.

To trigger up PSO, certain parameters should be defined. A Selection of these parameters, decides to a great extent (6) the ability of global minimization.

Population size $=100$

Number of iterations $=100$

Velocity constant, $\mathrm{c} 1=1.2$

Velocity constant, $\mathrm{c} 2=2$

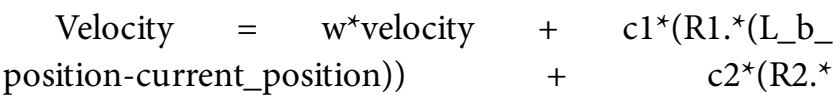
(g_b_position-current_position))

\subsection{Setpoint Tracking}

The setpoint variations have been brought in for evaluating the tracking potential of the projected PSO based PID controller (PSO-PID) and ERRC technique for the proposed systems, $G_{1}(s), G_{2}(s)$ and $G_{3}(s)$. Considering the cases: On the basis of considering time domain specifications, such as rise, and settling moment in time and a acceptable amount of overshoot and error response. ERRC implementation response yields superior results than PSO-PID control action. Results present in Figure 4-6, IMC-PID control is implemented and tested, the addition of ERRC with the designed controller, which disturbs the process variable and helps it to steady state at set point value with least amount rise time $\left(\mathrm{T}_{\mathrm{r}}\right)$ and settling time $\left(\mathrm{T}_{\mathrm{s}}\right)$, and acceptable over a broad range of process excess action. 

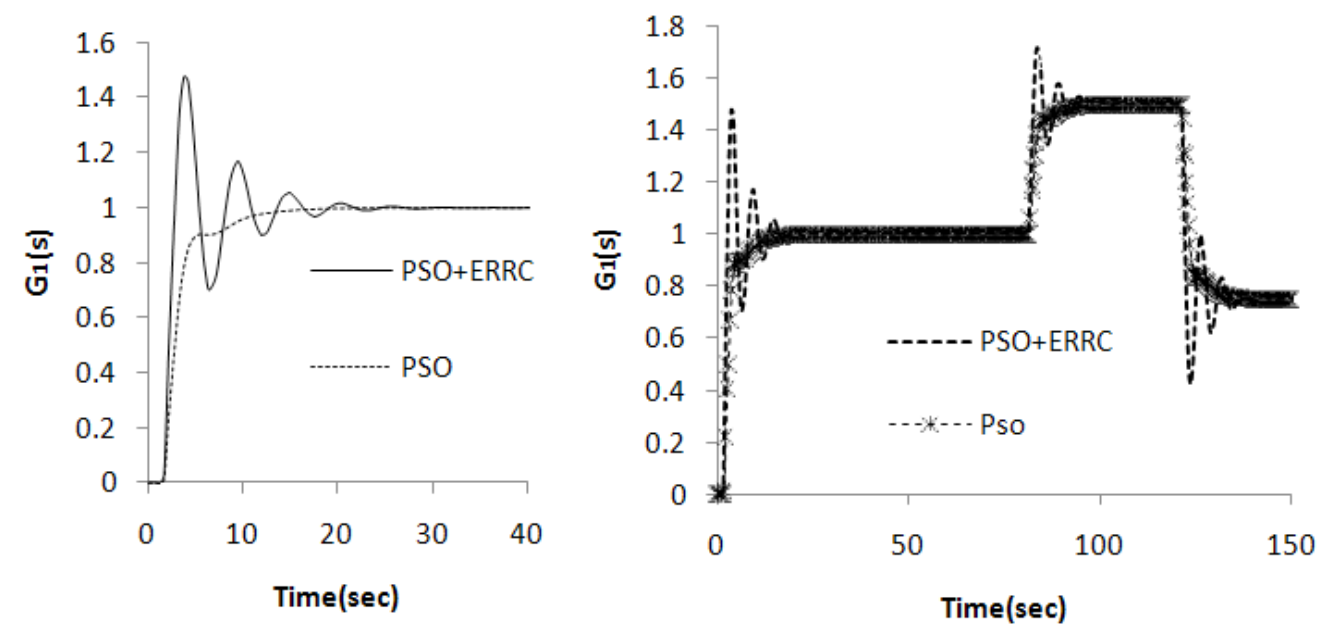

Figure 4. Set value tracking response of $G_{1}(s)$.
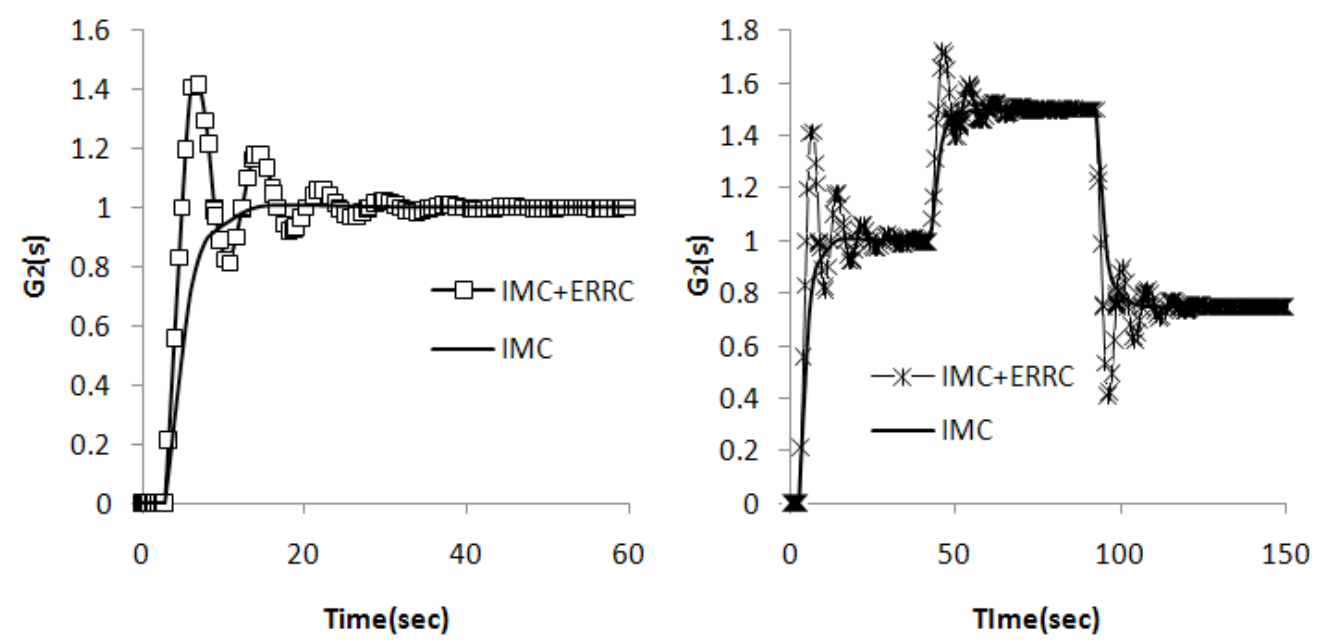

Figure 5. Set value tracking response of $\mathrm{G}_{2}(\mathrm{~s})$.
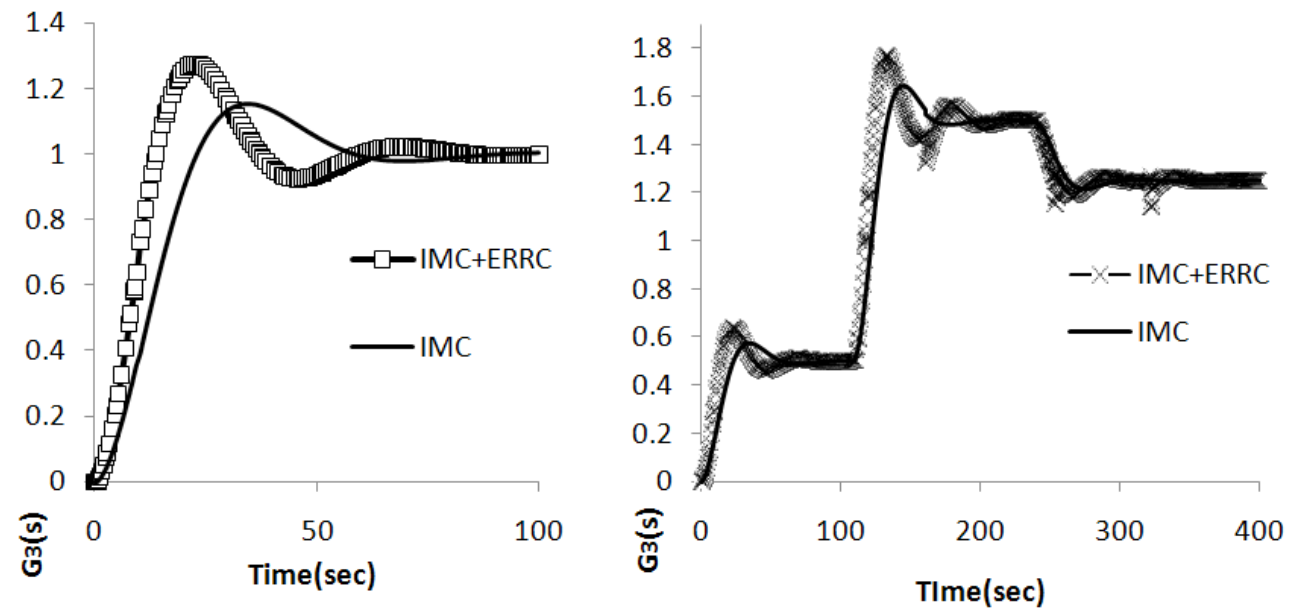

Figure 6. Set value tracking response of $\mathrm{G}_{3}(\mathrm{~s})$. 
Table 2. Gain values of tuned controller

\begin{tabular}{|l|l|l|l|l|l|}
\hline $\begin{array}{l}\text { S. } \\
\text { No. }\end{array}$ & System & $\begin{array}{l}\text { Control } \\
\text { Method }\end{array}$ & $\begin{array}{l}\text { Proportional Gain } \\
\left(\mathbf{K}_{\mathrm{p}}\right)\end{array}$ & $\begin{array}{l}\text { Integral Gain } \\
\left(\mathbf{K}_{\mathbf{i}}\right)\end{array}$ & $\begin{array}{l}\text { Derivative Gain } \\
\left(\mathbf{K}_{\mathbf{d}}\right)\end{array}$ \\
\hline 1 & $\mathrm{G}_{1}(\mathrm{~s})$ & PSO & 0.4173 & 0.1895 & 0.0779 \\
\hline 2 & $\mathrm{G}_{2}(\mathrm{~s})$ & IMC & 0.1876 & 0.0353 & 0.109929 \\
\hline 3 & $\mathrm{G}_{3}(\mathrm{~s})$ & IMC & 0.33 & 0.3173 & 1.0217 \\
\hline
\end{tabular}

\section{Conclusion}

In this study, the novelists of proposed ERRC method have projected and works for systems irrespective of system order, which displays critical variation in rise time and settling time. For the broad simulation learning, can be reasoned that the projected controller has a great set-point following, unsettling influence dismissal (disturbance) abilities at nominal operating points. An examination has come up with a winding up that the time domain specifications are lessened definitely for proposed technique than other tuning strategies.

\section{Reference}

1. Optimum setting for Automatic Controllers. Available from: https://staff.guilan.ac.ir/staff/users/chaibakhsh/ fckeditor_repo/file/documents/Optimum\%20Settings\%20 for\%20Automatic\%20Controllers\%20(Ziegler\%20and\%20 Nichols,\%201942).pdf
2. Theoretical consideration of retarded control. Availablefrom: https://www.scirp.org/(S(i43dyn45teexjx455qlt3d2q))/reference/ReferencesPapers.aspx?ReferenceID $=125717$

3. Astrom KJ, Hagglund T. Automatic tuning of simple regulators with specifications on phase and amplitude margins.Automatica. 1984; 20(5):645-51. https://doi.org/10.1016/0005-1098(84)90014-1

4. Robust process control. Available from: https://www. amazon.com/Robust-Process-Control-Manfred-Morari/ $\mathrm{dp} / 0137821530$

5. Generalised nonlinear PID controller based on neural networks. Available from: https://ieeexplore.ieee.org/document/754210

6. Genetic Algorithms for Level Control in a Real time Process. Available from: https://www.researchgate.net/ publication/237585781_Genetic_Algorithms_for_Level_ Control_in_a_Real_Time_Process

7. A fuzzy control scheme for nonlinear process. Available from: https://ieeexplore.ieee.org/document/6216193

8. IMC based design of PI controller for real time pressure process. Available from: https://www.researchgate. net/publication/294732252_IMC_Based_Design_of_PI_ Controller_for_Real_Time_Pressure_Process 